\title{
Influence of Guzheng Music Art, Music Theory thoughts of Han and Wei Dynasty on Later Generations
}

\author{
Jing Zhang \\ Xi'an Shiyou University, Xi'an Shaanxi, 710065, China
}

Keywords: Qin Guzheng; music theory thought; Qin Guzheng back to Qin

\begin{abstract}
Han and Wei dynasties are the periods with great development of Chinese economy and culture. Music culture was flourishing as never before. Guzheng as a Chinese ancient music instrument has obtained substantial development and received popularities by people in all social classes. It has become a music well favored and indispensable musical instrument. With the increase of its position, it has become an important accompaniment of musical instruments in Han Yuefu and Xianghe song. This paper tries to organize the Guzheng music art, and music theory thoughts in Han and Wei dynasties, and discuss its influence on later generations.
\end{abstract}

\section{Introduction}

The Han, Wei, and Six Dynasties are very important period in the development of our country's history. Musical culture in this period was connected to the early Qin Dynasty the late Sui and Tang dynasties. As an ancient musical instrument, Qin Guzheng was always remembered by people because of the retreat of playing Guzheng. Instead, it was favored by people of all walks of life from the emperor to the officials, from the government to common people, from scholars to the slaves and streetwalkers, from the town to the countryside as one of the most popular musical instruments. With the extinction of the pre-Qin Yayue, elegance and vulgarity can be treated dialectically in various stages of the Han and Wei period. As a musical instrument enjoying both elegance and popularity, Guzheng plays a unique artistic role with its rich expressiveness and beautiful timbres. It has become one of the important musical instruments of Han Yuefu. During this period, a large number of Qin Guzheng artists emerged who had exquisite skills and also wrote a lot of Guzheng fu, promoting Qin Guzheng and music theory thought to develop rapidly.

\section{Artists' music thoughts in Han and Wei dynasties}

\subsection{Artists' music thoughts in Han dynasties}

The Han Empire as a strong power had obtained unprecedented development in all aspects of politics and economy. The prosperity and national fusion has promoted the country to develop and flourish. A large-scale of Han Yuefu institution has emerged in this period in music field, which has created good conditions for mutual promotion of music among various nationalities and music recording and dissemination, thus producing a wide variety of musical forms.

The musical aesthetic thought in this period is the inheritance and development of Confucianism in the music field. In the process of music aesthetics, it emphasizes the function of practicality and enlightenment, establishes a music implementation process of "move people with emotions", and provides materialistic explanation for the basic musical aesthetic issue that music can express people's emotions, and it lays foundation for the idea of "music" and "political communication". As music can affect people's emotions and different kinds of music will make people feel differently, and then they will have appropriate ideas and behaviors. Therefore, music has the function of enlightenment, and has a decisive influence on social morality. As Hou Jin stated, music "can make people kind, have a deep impression on people, and it is easy to change the customs”. 
Music in this period emphasizes its political and moral standards, i. e, "Virtue is the first and prior than arts". Music with the ideological virtues in line with feudal order and ethics can be called finest works. Art standards are in the second place in this sense. However, it is not considered that "artistic" is irrelevant, and "morality" must be "decorated". Instead, it must be expressed in aesthetic form to bring people pleasure. So music can affect people's "goodness" and play a social role in cultivating people's virtues and changing social customs.

\subsection{Artists' music thoughts in Wei, Jin, Northern and Southern Dynasties}

In the end of the Eastern Han Dynasty, consorts and eunuchs alternated to grab all power, imperial power collapsed, and political condition was chaotic, which led to Dong Zhuo's abolition of the emperor. Han Empire collapsed completely. Over the following four hundred years, the country was in turmoil and political darkness. Due to frequent wars of change and endless wars, the scholars and the people fell into chaos. The serious disasters of the country and the people's suffering conditon had a profound influence on the art in this period and formed an aesthetic style centered on "sadness" as the beauty. This aesthetic intention has been described in many classics. It seems to emphasize that only grief can touch people's mind, indicating that there was objectively a sense of euphoria. The aesthetic imagination embodies a special state of mind in which the literary class were in a sad world and unable to transcend the grief and pain. Thus they resorted to helpless transcendence by virtue of their self-esteem and their mournful sorrows. The painful situation and inner grief forced the scholars to stay away from the artistic aesthetics of Confucian pragmatism and gradually approached the Laozi and Zhuangzi thoughts. "Appreciate in a super-practical taste to achieve the goals; obtain the judgement of human ethnics by thinking about aesthetics." Metaphysics in the Wei and Jin Dynasties emerged since thin. During this period, people's conscious awareness of artistic spirit has improved, which has exerted great influence on artistic creation and appreciation. It has sparkled with dazzling brilliance in the history of Chinese art. Qin Guzheng, as a musical instrument expressing a bitter sound, has become a kind of artifact loved by people to convey their thoughts and emotions.

\section{Music theory in Han and Wei dynasties}

In this period, Ruan Ji's "Views on Music" embodies the fusion between Confucianism and Taoism, and blended the aesthetic ideas of "the Confucian ethical code" and "nature." On one hand, it inherits the essential properties of Confucian philosophy of music and education, and regards the music in Zheng and Wei as obscene, requires "the song to be as good to be proper and to establish the sound of peace", to make ritual system, so as to "make musicians in the world abide by the ceremony", and emphasize the enlightening function of music.

Ji Kang in the same period put forward the slogan of "beyond the Confucian ethical code and let things in the course of nature." Which shows that "not an external, limited superficial work or activity, it should be a spirit, style, and style with infinite potential, thus to become a philosophy."(2). Ji Kang's "There is no sadness and joy in music" is a representative work of the aesthetic thought of music at that time. Its influence on contemporary and future generations cannot be ignored. Liu Sixie commented on this, "Teachers have unique opinions with delicate and innovative ideas". To oppose Confucianism's political practicality in the field of musical aesthetics, Ji Kang proposed "the harmony of the heart and the sound between both" Music is the objective sound, sorrow and joy are people's spiritual feeling after being touched. There exists no causal relationship, which reflects the idea of advocating music separated from feudal political utilitarianism and the music idea of advocating "ritual, music, punishment, and politics".

Ji Kang thinks that Guzheng is "quiet and loud, changeable and suitable for festivals." It shows that Guzhen has the tone characteristics that the sound is bright and varied, and the rhythm is fast. But his summary cannot cover all the characteristics of Guzheng as it can also express low and slow-pace music. In many books by Ruan Yu, Tao Rong's wife Chen, and Jia Bin, Hou Jin, and “Guzheng Fu”, the features of high and low tone, fast and slow speed, and variable rhythm are stated. According to these historical records, we can know that Guzheng can not only express delicate and subtle rhythms, 
but also can give out fluent sounds. It can be unworldly, popular and entertaining. These viewpoints have gather the aesthetic concepts of Confucianism, which is exactly a republication of Confucianism that "joyous but not indecent, mournful but not distressing," to lay the foundation of aesthetic thinking based on "virtue music". With "virtue music" as beauty, it has ethical and moral social attributes, and also requires the esthete to realize certain knowledge of rational content. With personal emotional attributes, namely, the emotional characteristic of happiness and pleasure, it also requires to carry out aesthetic sensing and aesthetic consciousness activities at the same time. Functions such as association and imagination should be used during aesthetic experience of music. Apart from emotional pleasure, it is also necessary to obtain rational an spiritual inspiration, which shows that GUzheng has the function of worship and entertainment, as well as the function of enlightenment, thus theoretically promote the social function to a new height.

There is no doubt that historical materials are always the same among thousands of people. Ji Kang thinks that Guzheng makes people "feel anxious in appearance but ambitious in heart" because the musical instruments "have big and small sound, and can impress people deeply or lightly". However, this effect only stops on the surface of people's emotions which cannot penetrate into people's inner feelings mainly because music is used to express people's thoughts. He believes that the noumenon of music is "harmony" and the sum of "size, single or complex, superior or inferior, good and evil". In other words, it is about the form of music, the means of expression, and the unity of beauty. Its role in appreciating viewers is limited to "quietness" and "specialization", namely, emotions or demureness in the emotional or sensory level, and the concentration or dispersal of spirits are congenital, physiological, which can be determined. There is no correlation between the lamentation and happiness. The emotional experience of funeral music is related to the experience of social life, cultural customs, etc. It is an emotion with certain social and cultural attributes. People's inner feelings are different, and understanding of music varies from person to person after being touched. Therefore, Ji Kang believes that music can arouse people's pleasant sense but it cannot play an enlightenment role of changing social customs. This has opposed the viewpoint of ignoring music artistry since two Han dynasties and equaling music to politics, witnessed the contradiction between music formal beauty, actual contents and appreciator's understanding. New ideas are proposed from the perspective of aesthetics.

\section{Influence of Han Wei Guzheng music art and music theory thoughts on later generation}

The reason why so many scholars like Guzheng is because it has a wide range of vocals, especially the timbre is bright and crisp. It can give out euphemistic and bitter music, and also can play high-tone passionate melody, especially by playing with the left hand. Reshaping various techniques can help to get close to people's hearts and bring resonance with people, give people lingering and appreciator feelings, evoke people's sad mood and then stimulate sad feelings in the heart, let off painful emotions, thus obtaining the aesthetic feeling just like tragedy appreciation. After the Wei and Jin Dynasties, music became more and more inclined to express grief. The "mourning” reflects various potential contradictions the accumulated, brewing and agitated in the feudal society during this period. It is an era full of twists and turns, hardships, and resentment in history. In the social soil of this social period, Wei Jin and the South and North Dynasties have ushered in the establishment and prosperity of the theory and music aesthetic system of Guzheng. From this point of view, the music emerged with compassion as virtue and people listening to music find their bosom friends due to the sad feelings, "Sadness is beauty" has become the aesthetic style in Han, Wei and Six dynasties. In Wang Bao’s "Dong Xiao Fu", Wang Chong's "Lun Heng Zi Ji", and Ma Rong's "Preface of Flute Fu", the description of "sad music is pleasant" can be seen. It seems that only grief can impress people, which shows people at time feel delighted to appreciating sad music, which is closely linked with the destiny of the country. All these are connected with the pessimistic and complex condition at that time. This increasingly conscious aesthetic pursuit was expressed through scholars' numerous poetry and lyrics, and accumulated repeatedly, thus finally conducting to the mental set of Guzheng aesthetics. The trend reached its peak in the Wei and Jin dynasties. It can be seen that the reason why 
people in ancient time showed special preference for Qin Guzheng is because its melody can perfectly impress people and infect people, arouse people's emotions and resonance. So it has become a good carrier for people to express feelings and emotions.

Since from Qin and Han dynasties, Guzheng gradually spread from the northwest region of China to the whole country, fused with local opera, rap music, and folk music to establish a variety of local genre. As the famous Zhejiang Guzheng facion master, Wang Xunzhi said, about the "Shaanxi faction focuses more on lyric.” Qin Guzheng as Qin school in Shaanxi, the style is delicate, with euphemism and resentment; the musical style of Guzheng is often associated with miserable musical emotions, such as Cen Shen's “Qin Zheng Song,” “Qin Gguzheng sounds the most miserable.” Liu Zhongyong said in "Listening to Guzheng" that "Play Guzheng and hear the infinite Qin people's grievances," Qin Guzheng is good at expressing this tune. Therefore, we can say Shaanxi Zheng faction has become the representative of a music genre.

Since from opening up and reform, Shaanxi Qin Guheng has actively advocated the academic concept of "Qin Guzheng back to Qin”, united with the Guzheng musicians in this area and learned from the folk people. According to Shaanxi local opera and folk music, a large number of adaptations and creations have emerged with a batch of research paper published, which is not only the specific practice of Qin Guzheng back to the Qin, but also the inheritance and development of the traditional Guzheng music. A large number of Guzheng music has become a popular repertoire in all kinds of competitions across the country, and it is widely recognized and loved by Guzheng lovers and the ordinary people such as "The Mulberry of Qin”, "Hometown Rhyme”, "Yun Sang Su” and "Wuling Yin”, "Jiangnv's Tears”. "Ginger female tears" and so on. The reason why so many people like this tragic melody is because it is beautiful and appealing, and it can impress people and make people resonate in their hearts. The infectious power embodies the inner grief and has significance of positive, constantly fighting, and unwilling to yield. In addition, the common feature of Guzheng music is to move and impress people with affection which includes emotions such as family affection, nostalgia, sentiment of missing, etc. The feeling is the emotional expression needed and desired in nowadays society. Because of the feature that Shaanxi Guzheng can fully develop the emotional feelings in people's heart vividly by playing with left hand metrically, we can say that it is precisely the most characteristic style of Shaanxi Qin Guzheng gener music.

\section{Summary}

The aesthetic taste of love from sadness and sorrow-oriented aesthetics have continued until now and obtained better development. It is influencing more and more people who love Guzheng and playing Guzheng. Guzheng music aesthetics in Wei, Jin, Northern and Southern Dynasties have profound significance on Guzheng professional education in modern system and aesthetics of Guzheng music art.

\section{References}

[1] Jiao Wenbin. “Historical view on Qin Guzheng” ,China Federation of Literary and Art Circles Publishing House, 2002.

[2] Wu Zhao, Liu Dongsheng, Brief History of Chinese Music ,People's Music Publishing House, 1983.

[3] Yang Yinliu, Brief History of Chinese Ancient Music ,People's Music Publishing House, 1980.

[4] Xiang Yang. History of Chinese Bowed and String Instrument ,Int'l Culture Publishing House, 1999.

[5] Liu Zaisheng, Brief discussion on Chinese ancient music history ,People's Music Publishing House, 1989. 
[6] Xiao Difei, Yuefu literature history in Han Wei and Six dynasties,People's Literature Publishing House, 1984.

[7] Li Zehou, “Three Books on Aesthetics”, Anhui Literature \& Art Press, 1999.

[8] Xiu Hailin, Luo Xiaoping, General Theory of Music Aesthetic, Shanghai Music Publishing House, 1999.

[9] Xu Fuguan, Chinesea Art Spirit,Chunguang Literature \& Art Press , 1987.

[10] Ji Liankang, Historical materials in Wei, Jin and Northern and Southern Dynasties, Shanghai Literature and Art Press ,1982.

[11] Xu Fuguan, “Chinese Art Spirit”,Chunguang Literature \& Art Press, June, 1987, page 130.

[12] Li Zehou, “Three Books on Aesthetics”, Anhui Literature \& Art Press, Jan, 1966, page 97. 